\title{
The CLIF Consortium Acute Decompensation score (CLIF-C ADs) for prognosis of hospitalised cirrhotic patients without acute-on-chronic liver failure
}

\author{
Rajiv Jalan ${ }^{1}$, Marco Pavesi $^{2}$, Faouzi Saliba ${ }^{3}$, Alex Amorós $^{2}$, Javier Fernandez ${ }^{4}$, \\ Peter Holland-Fischer ${ }^{1}$, Rohit Sawhney ${ }^{1}$, Rajeshwar Mookerjee ${ }^{1}$, Paolo Caraceni ${ }^{5}$, \\ Richard Moreau $^{6}$, Pere Ginès ${ }^{4, *}$, Francois Durand ${ }^{3}$, Paolo Angeli ${ }^{7}$, Carlo Alessandria ${ }^{8}$, \\ Wim Laleman ${ }^{9}$, Jonel Trebicka ${ }^{10}$, Didier Samuel ${ }^{3}$, Stefan Zeuzem ${ }^{11}$, Thierry Gustot ${ }^{12}$, \\ Alexander L. Gerbes ${ }^{13}$, Julia Wendon ${ }^{14}$, Mauro Bernardi ${ }^{15}$, Vicente Arroyo ${ }^{15}$, \\ CANONIC Study Investigators; EASL-CLIF Consortium ${ }^{\dagger}$
}

${ }^{1}$ Liver Failure Group, Institute for Liver and Digestive Health, Royal Free Hospital, London, United Kingdom; ${ }^{2}$ Data Management Center, EASL-CLIF Consortium, Barcelona, Spain; ${ }^{3}$ Hôpital Paul Brousse, Villejuif, France; ${ }^{4}$ Hospital Clínic, University of Barcelona,

IDIBAPS, CIBEReHD, IRSIN, Spain; ${ }^{5}$ Policlinico St Orsola Malpighi, Bologna, Italy; ${ }^{6}$ Hopital Beaujon, Clichy, France; ${ }^{7}$ University of Padua, Padova, Italy; ${ }^{8}$ University of Turin, Torino, Italy; ${ }^{9}$ University Hospital Gasthuisberg, Leuven, Belgium; ${ }^{10}$ University Hospital Bonn, Bonn, Germany; ${ }^{11}$ J.W Goethe University Hospital, Frankfurt, Germany; ${ }^{12}$ Erasme Hospital Brussels, Brussels, Belgium; ${ }^{13}$ University of Munich, Klinikum der LMU, Munich, Germany; ${ }^{14}$ King's College Hospital, London, United Kingdom; ${ }^{15}$ EASL-CLIF Consortium, Barcelona, Spain

Background \& Aims: Cirrhotic patients with acute decompensation frequently develop acute-on-chronic liver failure (ACLF), which is associated with high mortality rates. Recently, a specific score for these patients has been developed using the CANONIC study database. The aims of this study were to develop and validate the CLIF-C AD score, a specific prognostic score for hospitalised cirrhotic patients with acute decompensation (AD), but without ACLF, and to compare this with the Child-Pugh, MELD, and MELD-Na scores.

Methods: The derivation set included 1016 CANONIC study patients without ACLF. Proportional hazards models considering liver transplantation as a competing risk were used to identify score parameters. Estimated coefficients were used as relative

Keywords: Acute-on-chronic liver failure; Hepatic encephalopathy; Chronic liver failure.

Received 23 July 2014; received in revised form 28 October 2014; accepted 4 November 2014; available online 22 November 2014

* Corresponding author. Address: Secretary of the EASL-CLIF CONSORTIUM, Liver Unit, Hospital Clinic, University of Barcelona, Catalonia, Spain. Tel.: +34 93 2271713; fax: +34 932271779

E-mail address: PGINES@clinic.ub.es (P. Ginès).

The complete list of CANONIC Study Investigators is reported at the end of the manuscript.

Abbreviations: ACLF, acute-on-chronic liver failure; AD, acute decompensation; CANONIC study, EASL-CLIF Acute on chroNIC liver failure study; CLIF, Chronic Liver Failure; CLIF-C ACLFs, CLIF-CONSORTIUM ACLF score; CLIF-C OFs, CLIFConsortium Organ Failure score; CLIF-SOFAs, CLIF-Sequential Organ Failure Assessment score; CPs, Child-Pugh score; E, epinephrine; EASL, European Association for the Study of the Liver; $\mathrm{FIO}_{2}$, fraction of inspired oxygen; HE hepatic encephalopathy; INR, International Normalized Ratio; MAP, mean arterial pressure; MELDs, Model of End-Stage Liver Disease; MELD-Nas, MELD-Sodium score; NE, norepinephrine; $\mathrm{PaO}_{2}$, partial pressure of arterial oxygen; SOFA Sequential Organ Failure Assessment; $\mathrm{SpO}_{2}$, pulse oximetric saturation. weights to compute the CLIF-C ADs. External validation was performed in 225 cirrhotic AD patients. CLIF-C ADs was also tested for sequential use.

Results: Age, serum sodium, white-cell count, creatinine and INR were selected as the best predictors of mortality. The C-index for prediction of mortality was better for CLIF-C ADs compared with Child-Pugh, MELD, and MELD-Nas at predicting 3- and 12-month mortality in the derivation, internal validation and the external dataset. CLIF-C ADs improved in its ability to predict 3-month mortality using data from days $2,3-7$, and $8-15$ (C-index: 0.72 , 0.75 , and 0.77 respectively).

Conclusions: The new CLIF-C ADs is more accurate than other liver scores in predicting prognosis in hospitalised cirrhotic patients without ACLF. CLIF-C ADs therefore may be used to identify a high-risk cohort for intensive management and a low-risk group that may be discharged early.

(c) 2015 European Association for the Study of the Liver. Published by Elsevier B.V. All rights reserved.

\section{Introduction}

Patients with cirrhosis who require admission to hospital with acute decompensation (AD) (ascites, gastrointestinal bleeding, hepatic encephalopathy and/or acute bacterial infections) have widely variable prognosis dependent on whether they have acute-on-chronic liver failure (ACLF) [1-3], which is diagnosed using the CLIF Consortium organ failure score (CLIF-C OFs) $[4,5]$. In the CANONIC study, which was performed in patients with $\mathrm{AD}$ of cirrhosis with and without ACLF, the 3-month mortality of patients with ACLF was 51\% [5]. A specific score to

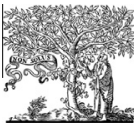

Journal of Hepatology 2015 vol. 62 | 831-840

ELSEVIER 


\section{Research Article}

predict prognosis in patients with ACLF, the CLIF-C ACLF score (CLIF-C ACLFs) has been developed [3].

In the CANONIC patients with AD who did not develop ACLF, the 28 -day mortality was $4.6 \%$, but this increased to $12.6 \%$ at 3 -month, $18.3 \%$ at 6 months, and $27.6 \%$ at 1 -year (AD patients) [5]. These data suggest that some patients with $\mathrm{AD}$ are also at high risk of short-term mortality; they should be recognised early and treated as potentially high-risk patients requiring closer monitoring and interventions to prevent progression to ACLF and death. On the other hand, patients with AD who are at low risk of mortality may be discharged early, potentially saving resources and distress for the patients and their relatives. At present a specific prognostic score focussing on $\mathrm{AD}$ patients is an unmet need $[2,6]$. Therefore, the main objective of this study was to develop a new score (CLIF-Consortium score for $A D$ patients, CLIF-C ADs) with a higher prognostic accuracy than the currently used scoring systems such as MELDs [7], MELD-Nas [8], and Child-Pugh score (CPs) [9]. The study had three main aims. First, to develop a scoring system for sequential use based on clinical and biochemical data to prognosticate survival of $A D$ patients who did not fulfil criteria for the diagnosis of ACLF (CLIF-C ADs) [5]. Second, to compare the prognostic accuracy of CLIF-C ADs with MELDs, MELD-Nas, and CPs. Third, to validate the prognostic accuracy of the CLIF-C ACLFs in two prospective external cohorts of hospitalized cirrhotic patients with $\mathrm{AD}$ and no ACLF. We used the CANONIC study database to develop this score because it had over 1000 prospectively included patients with AD that were followed for 1-year in multiple centres in Europe. The validation sets were drawn from hospitals in Barcelona (Hospital Clinic) and London (Royal Free Hospital).

\section{Materials and methods}

\section{Study populations}

The study was performed in cirrhotic patients with $A D$ from two different populations. The CANONIC study population included 1349 cirrhotic patients from 29 European hospitals who had developed AD leading to hospitalization, and were prospectively followed-up to 1-year. Reasons for exclusion were: patients with decompensated cirrhosis admitted for a scheduled procedure or treatment, patients with hepatocellular carcinoma outside Milan criteria, severe chronic extra-hepatic disease, HIV infection or immunosuppressive therapy, and patients who refused to participate [5]. Ethical review boards in individual countries approved the study [5]. The CANONIC patients who did not develop an ACLF episode at enrollment and had all the data required to compute the studied scores at study enrollment were included in the score derivation set [5]. The population used for external validation consisted of 225 patients admitted to two European hospitals with AD of cirrhosis and not presenting an ACLF episode.

\section{Statistical methods}

All the variables used in statistical analyses were obtained at the time of study enrollment, which coincided with hospital admission in most patients (98\%). In the CANONIC series, a simplification of the CLIF-SOFA score [10] named CLIF-C OF score, was used to diagnose ACLF and classify patients based on severity. CLIF-C OF score included 6 sub-scores - one for each organ/system (liver, kidney, brain, coagulation, circulation, and respiration) - each of them ranging from 1 to 3 with an aggregate score ranging 6-18. The categories included in CLIF-C OF score sub-scores and the corresponding cut-off values were derived from a consensus and the aggregate score proved to accurately predict mortality and ACLF onset in patients with $\mathrm{AD}[3,5]$.
With the purpose of deriving a simple, specific prognostic score for cirrhotic patients with $A D$ and without ACLF that would only include objective clinically relevant predictors, we assessed the association between each of the patients' clinical characteristics and laboratory parameters at study enrollment and the mortality observed at each main time-point (28, 90, 180, and 365 days). The objective was to select a limited group of predictors related to both short and long-term mortality with a reduced co-linearity. Score parameters were then to be chosen among these predictors. To assess if the predictive ability of CLIF-C $\mathrm{AD}$ score could be improved by clinical subjective parameters, such as ascites or encephalopathy, all factors significantly associated with mortality in the univariate analysis and not selected for the final score model were individually added to the model, testing the change in the corresponding C-index. In all univariate statistical comparisons, the Chi-square test was used for categorical variables, Student's $t$ test and Mann-Whitney tests for continuous variables. McNemar test and paired $t$ test were used to compare repeated measurements of categorical and continuous variables, respectively. Among the CANONIC patients with AD and without ACLF, 160 Patients (15.7\%) underwent a liver transplantation within 1 year after their hospitalization, 76 in the first 3 months (7.5\%), 40 between 3 and 6 months (3.9\%), and 44 (4.3\%) after 6 months. As the study was performed in many centres around Europe, there was no agreed prioritization for transplantation. The decision to transplant a particular patient or not, and whether they were prioritized was decided by individual centres. So, in order to identify the main predictors for mortality and to estimate the corresponding effects adjusting for the impact of liver transplantation as well as for the geographic differences in transplant dynamics, a proportional-hazards model considering liver transplantation as a factor "competing" with mortality risk was used to select score parameteres [11,12]. The group of baseline factors significantly $(p<0.05)$ associated with mortality at main study time-points and with a limited internal co-linearity (pairwise Pearson's correlation coefficients of 0.5 or lower) were used to fit the final model. PH-CR models including all the selected factors were fitted applying a forward step-wise selection method with $p$-in $=0.05$ and $p$ out $=0.1$. The coefficients estimated for each factor in the 90-day model, which provided the best predictive ability, were used as relative weights to compute the CLIF-C AD score.

The calibration of CLIF-C AD score was assessed by comparing the actual observed risk and the average probability of dying at different time-points predicted by the score. The observed and predicted probabilities were also compared across different levels of CLIF-C AD score by means of the Hosmer-Lemeshow $\chi^{2}$ test to assess the corresponding goodness-of-fit.

Harrell's concordance index (C-index) was used to assess the score discrimination ability $[13,14]$. Since a PH-CR model was used, C-index values and the corresponding $95 \%$ confidence intervals (Cl's) were estimated treating the transplanted patients as censored at the end of the follow-up, assuming that none of them could die before [11]. Statistical comparisons of C-index between CLIF-C AD, CLIF-OF, MELD, MELD-Sodium and CPs were carried out for the main study time-points using the Integrated Discriminating Improvement (IDI) statistic [14]. A confirmatory analysis was carried out to assess the discrimination ability of CLIF-C ADs and of the other scores by estimating and comparing the corresponding Areas Under the ROC curves (AUROCs) for the 90-day mortality endpoint, which was used to fit the final score model [15].

Internal and external validation of the CLIF-AD score

Both an internal and an external validation of CLIF-C AD score were carried out [16]. A sample of 500 patients randomly selected from the CANONIC non-ACLF population was used to internally validate the CLIF-C AD score comparing the C-index estimates with those obtained for MELDs, MELD-NAs and CPs by means of the same methods applied to the CANONIC data. The external validation was carried out applying the same statistical techniques as for the internal validation to an external sample of 225 patients hospitalized for an AD of cirrhosis without ACLF and showing similar clinical characteristics as the CANONIC patients. Patients included in this external validation set came from prospectively collected data from two different European centers, the Royal Free Hospital, London (97 patients) and the Hospital Clinic, Barcelona (128 patients).

Validation of the CLIF-AD score for sequential use

The prognostic ability of CLIF-C AD and MELD scores for sequential use was assessed using the sub-set of CANONIC patients without ACLF at enrollment and data available at the time of enrollment, at $48 \mathrm{~h}$, at 3-7 days, and at 815 days after enrollment. Post-enrollment C-indexes for 1 -year mortality were compared with baseline (enrollment) by means of paired $t$ tests. 


\section{JOURNAL OF HEPATOLOGY}

\section{Results}

Study populations

The derivation set included 1016 of the 1349 CANONIC study patients who did not present ACLF at study enrolment and had all the data required to compute the studied scores $[3,5]$. The etiology of cirrhosis was mainly alcoholic or chronic hepatitis $C$ ( $80 \%$, Table 1$)$. In the remaining patients, the causes of cirrhosis were chronic hepatitis B (67 patients, 6.6\%), cryptogenic (61, $6.0 \%)$, NASH $(55,5.4 \%)$, primary biliary cirrhosis $(24,2.4 \%)$ and other causes. Among the 67 patients with HBV, 29 (43.3\%) were receiving treatment with anti-viral drugs within 3 months prior to enrollment: 13 (19.4\%) received Entecavir, 10 (14.9\%) Tenofovir, 4 (6.0\%) Lamivudine (3 alone and one in combination with Tenofovir), and 2 (3.0\%) Telbivudine (one alone and one in combination with Entecavir). The database used for external validation included 225 non-ACLF patients from two centres. Etiology of cirrhosis in these patients was similar to that in the derivation set. All patients from both the derivation and the external validation cohorts were followed-up for a year. Criteria for the inclusion/exclusion in the validation set were those applied to the CANONIC study. Patients in the external validation cohort were more frequently male, had a higher rate of hepatic encephalopathy grade III-IV, higher levels of white blood cells and INR than the CANONIC cohort. The use of vasopressors was significantly higher in the derivation set. However, the rest of clinical and laboratory parameters at admission as well as MELDs, MELDNas and CPs and mortality rates were similar in the derivation and validation sets, except for 1-year mortality rate, which was higher in the CANONIC cohort (Table 1).

\section{Development of the CLIF-C ADs}

The new CLIF-C AD score was developed with the purpose of identifying a limited number of independent predictors. Therefore, univariate analyses compared survivors and non-survivors at the main study end-points (mortality rates at 90,180, and 365 days) including all the parameters used to compute the CLIF-C OFs as well as all clinical characteristics and laboratory parameters at patients' enrollment. The 28-day mortality was very low (4\%, 47 patients). Therefore, this was not taken into account as the main outcome for multivariate modelling. The baseline factors significantly associated with short- and long-term mortality were age, ascites (clinically diagnosed), gastrointestinal-bleeding, serum sodium, creatinine, INR, serum potassium, white cell count and C-reactive protein (Supplementary Table 1). After fitting an initial CR-PH model with all these factors, age, serum sodium and log-transformed white cell count, creatinine and INR were selected as the best predictors.

Table 1. Baseline characteristics and outcome of patients without an Acute-on-Chronic Liver Failure (NO ACLF) in the CANONIC study and in the external validation datasets.

\begin{tabular}{|c|c|c|c|}
\hline Patients' characteristics & Derivation set (CANONIC patients) $(n=1016)$ & External validation set $(\mathrm{N}=225)$ & $p$ value \\
\hline Age (years) & $57.6 \pm 12.4$ & $54.9 \pm 10.7$ & 0.001 \\
\hline Male sex & $638(62.8)$ & $159(71.0 \%)$ & 0.031 \\
\hline \multicolumn{4}{|c|}{ Patients' characteristics at study enrollment } \\
\hline \multicolumn{4}{|c|}{ Etiology of cirrhosis: } \\
\hline Alcohol & $468(49.0)$ & $110(48.9)$ & 0.461 \\
\hline $\mathrm{HCV}$ & $207(21.7)$ & $41(18.2)$ & 0.519 \\
\hline Alcohol + HCV & $92(9.6)$ & $33(14.7)$ & $<0.001$ \\
\hline Presence of ascites & $646(63.9)$ & $148(65.8)$ & 0.591 \\
\hline Serum bilirubin $(\mu \mathrm{mol} / \mathrm{L})$ & $79 \pm 95$ & $68 \pm 59$ & 0.096 \\
\hline Serum creatinine $(\mu \mathrm{mol} / \mathrm{L})$ & $85 \pm 32$ & $81 \pm 29$ & 0.085 \\
\hline HE grade I-II & $255(25.1)$ & $37(16.4)$ & 0.007 \\
\hline HE grade III-IV & $23(2.3)$ & $18(8.0)$ & 0.001 \\
\hline Intern. normalised ratio & $1.5 \pm 0.4$ & $1.6 \pm 0.3$ & $<0.001$ \\
\hline Serum sodium (mmol/L) & $135 \pm 6$ & $135 \pm 5.5$ & 0.999 \\
\hline White cell count $\left(x 10^{9}\right.$ cells/L) & $6.8 \pm 4.1$ & $7.6 \pm 4.8$ & 0.021 \\
\hline Use of vasopressors & $159(15.7)$ & $5(2.2)$ & $<0.001$ \\
\hline $\mathrm{PaO}_{2} / \mathrm{FiO}_{2} \leq 200$ or $\mathrm{SpO}_{2} / \mathrm{FiO}_{2} \leq 214$ & $7(0.8)$ & $3(1.3)$ & 0.572 \\
\hline \multicolumn{4}{|l|}{ Scores at study enrollment } \\
\hline MELD score & $16 \pm 5$ & $16 \pm 5$ & 0.999 \\
\hline MELD-Na Score & $19 \pm 6$ & $19 \pm 5$ & 0.999 \\
\hline Child-Pugh Score & $9.3 \pm 2.0$ & $9.1 \pm 1.9$ & 0.171 \\
\hline \multicolumn{4}{|l|}{ Mortality rates } \\
\hline 28-day mortality & $47(4.6)$ & $10(4.4)$ & 0.954 \\
\hline 90-day mortality & $128(12.6)$ & $22(9.8)$ & 0.260 \\
\hline 6-month mortality & $186(18.3)$ & $37(16.4)$ & 0.565 \\
\hline 1-year mortality & $280(27.6)$ & $47(21.0)$ & 0.045 \\
\hline
\end{tabular}

Data are numbers of patients (\%) or mean \pm SD.

$\mathrm{HE}$, hepatic encephalopathy; HRS, hepato-renal syndrome; $\mathrm{FIO}_{2}$, fraction of inspired oxygen; $\mathrm{PaO}_{2}$, partial pressure of arterial oxygen; SpO ${ }_{2}$, pulse oximetric saturation. 


\section{Research Article}

Surprisingly, bilirubin was not included in the subset of the best predictors: this was probably due to the smaller effect-size observed at the different time-points (Fig. 1).

The CLIF-C ADs was computed by applying model coefficients. Although in our series the extreme values were from 23.4 to 81.6 , the potential values in some patients might exceed these limits and be negative or higher than 100 . The score was then trimmed between 0 and 100, since lower and upper values did not modify the expected probabilities of dying by more than $1 \%$. In the whole series the mean CLIF-C ADs was 51.2 (SD: 8.7). Supplementary Fig. 1 shows the CLIF-C ADs histogram in our series.

The equation for CLIF-C ADs is:

$$
\begin{aligned}
\text { CLIF-C ADs }= & 10^{*} 0.03^{*} \text { Age }\{\text { years }\}+0.66^{*} \operatorname{Ln}(\text { Creatinine }\{\mathrm{mg} / \mathrm{dl}\}) \\
& +1.71^{*} \operatorname{Ln}(\mathrm{INR})+0.88^{*} \operatorname{Ln}\left(\mathrm{WBC}\left\{10^{9} \text { cells } / \mathrm{L}\right\}\right) \\
& -0.05^{*} \operatorname{Sodium}\{\mathrm{mmol} / \mathrm{L}\}+8
\end{aligned}
$$

The probability of death at time " $\mathrm{t}$ " was estimated by the equation:

$p=1-\mathrm{e}^{\left(-\mathrm{C}(\mathrm{t})^{*} \exp \left(\beta(\mathrm{t})^{*} \mathrm{CLIF}-\mathrm{C} \mathrm{ADs}\right)\right)}$

$\mathrm{CI}(\mathrm{t})$ and $\beta(\mathrm{t})$ are the cumulated baseline hazard and the score coefficient estimated by the model fitted for time $t$. At the main time-points they are: $\mathrm{CI}(90)=0.00056$, $\beta(90)=0.1007 ; \quad \mathrm{Cl}(180)=0.00173, \quad \beta(180)=0.0889 ; \quad \mathrm{CI}(365)=$ $0.00879, \beta(365)=0.0698$.

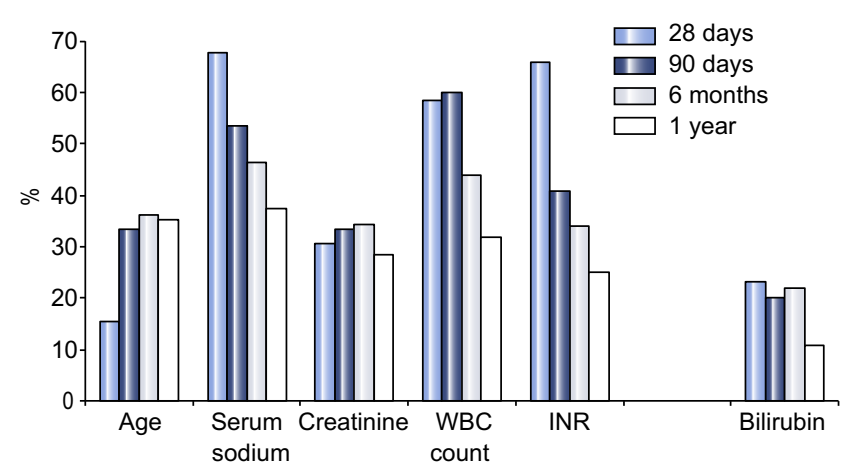

Fig. 1. Percentage effect-size (mean difference between alive and dead patients/standard deviation) for the main predictors of mortality in univariate analysis.
An online application to estimate the predicted death rate at time ' $\mathrm{t}$ ' based on CLIF-C ADs is available at the CLIF-Consortium website: http://www.clifconsortium.com/.

\section{Calibration of the CLIF-C ADs}

The predicted and observed probabilities of death at 1-year were similar across the different levels of CLIF-C ADs (Supplementary Fig. 2): Hosmer-Lemeshow $\chi^{2}=13.1, p=0.12$ ). The probabilities of death estimated for the mean value of CLIF-C ADs were almost identical to the overall mortality rates observed at all main study time-points: 90 -day (0.12 vs. 0.13$), 6$-month (0.19 vs. 0.18$)$, and 1 -year (0.31 vs. 0.28).

Discrimination ability of CLIF-C ADs. Comparison with CLIF-OFs, MELDs, MELD-Nas, and CPS

The C-Index of CLIF-C ADs for 28-day, 90-day, 6-month, and 1 -year mortality $(0.75,0.74,0.71$, and 0.67$)$ were significantly better than those corresponding to CLIF-C OFs $(0.68$ [ $p<0.001]$, 0.61 [ $p<0.001$ ], 0.58 [ $p<0.001$ ], and 0.56 [ $p<0.001])$. CLIF-C ADs significantly improved the predictive discrimination of all other scores at 90 days and on longer term (Table 2). In particular, the improvements of 6 to 10 points in C-index values with respect to MELDs were consistently significant at all time-points. Fig. 2 shows the corresponding percentage improvement

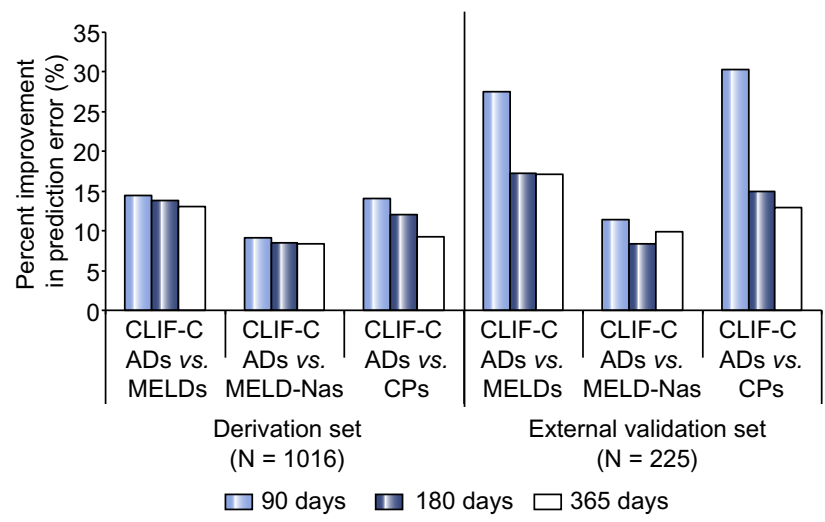

Fig. 2. Relative (percentage) reduction in prediction error rates of CLIF-C AD

\begin{tabular}{|c|c|c|c|c|}
\hline & $\begin{array}{l}\text { CLIF-C AD score } \\
\text { C-index }(95 \% \mathrm{Cl})\end{array}$ & $\begin{array}{l}\text { Child-Pugh score } \\
\text { C-index }(95 \% \mathrm{Cl})\end{array}$ & $\begin{array}{l}\text { MELD score } \\
\text { C-index }(95 \% \mathrm{Cl})\end{array}$ & $\begin{array}{l}\text { MELD-Na score } \\
\text { C-index }(95 \% \mathrm{Cl})\end{array}$ \\
\hline \multicolumn{5}{|c|}{ CANONIC patients $(\mathrm{N}=1016)$} \\
\hline 90-day mortality & $0.743(0.704-0.783)$ & $0.651(0.601-0.701)$ & $0.649(0.602-0.697)$ & $0.681(0.633-0.728)$ \\
\hline$p$ value vs. CLIF-C ADs* & & $<0.001$ & $<0.001$ & $<0.001$ \\
\hline 180-day mortality & $0.711(0.675-0.747)$ & $0.635(0.593-0.677)$ & $0.625(0.585-0.665)$ & $0.655(0.615-0.695)$ \\
\hline$p$ value vs. CLIF-C ADs * & & $<0.001$ & $<0.001$ & $<0.001$ \\
\hline 365-day mortality & $0.670(0.639-0.702)$ & $0.613(0.578-0.648)$ & $0.593(0.560-0.627)$ & $0.618(0.584-0.652)$ \\
\hline$p$ value vs. CLIF-C ADs * & & $<0.001$ & $<0.001$ & $<0.001$ \\
\hline
\end{tabular}
score as compared to Child-Pugh (CP), MELD, and MELD-Na scores.

Table 2. Predictive discrimination ability of CLIF-C AD score as compared to MELD and MELD-Sodium. Patients without ACLF at enrolment in CANONIC database.

${ }^{*} p$ values from the Integrated Discriminating Improvement (IDI) statistics test. 


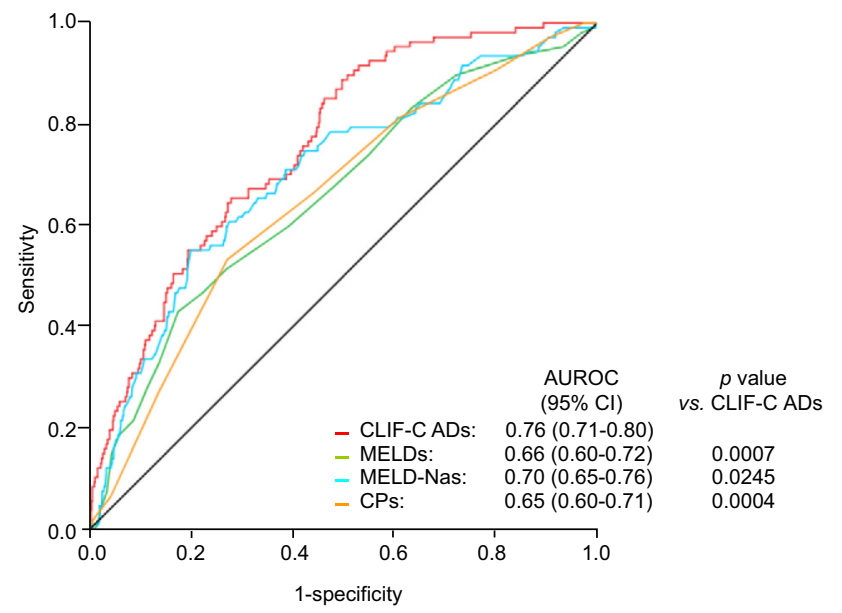

Fig. 3. Predictive ability of CLIF-C AD score for 90-day mortality as compared to MELD, MELD-Na and Child-Pugh (CP) score.

obtained with CLIF-C ADs in prediction error rate with respect to the other scores (computed as percentage reduction in discordance rate of CLIF-C ADs vs. Reference score, i.e. $100 \times$ [C-in-

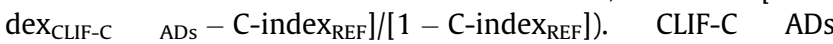
consistently improved the prediction error rates observed for MELDs and CPs (the relative improvements ranged between $8 \%$ and $14.5 \%$ at the different time-points). The percentage reductions in discordance rates of CLIF-C ADs as compared to those observed with MELD-Nas were lower (between $4 \%$ and $9 \%$ ), but still consistent across all time-points.

The analysis carried out by comparing the AUROCs corresponding to CLIF-C AD score, MELD, MELD-Na, and CP score for 90-day confirmed the superiority of CLIF-C ADs and the improvement in predictive ability with respect to the other scores (Fig. 3).

We explored different cut-off values of CLIF-C ADs potentially useful to discriminate the sub-groups of patients at the lowest and highest risk of dying. In the 274 patients $(27.0 \%)$ with a CLIF-C ADs equal to or lower than 45, 90-day mortality rate (1.8\%, $95 \% \mathrm{CI}: 0.8 \%-4.2 \%)$ was 5 times lower than in whole series

\section{JOURNAL OF HEPATOLOGY}

of $\mathrm{AD}$ patients. The corresponding survival at 6 (94.2\%; $95 \% \mathrm{CI}$ : 90.7\%-97.4\%) and 12 months (85.4\%; 95\% CI: 80.7\%-89.1\%) were also very high. On the other hand, a group of 163 patients (16\%) with a score of 60 or higher presented a 3-fold increase as compared to the overall mortality rates. Their mortality at 3 , 6, and 12 months was 31.3\% (95\% CI: $24.7 \%-38.8 \%$ ), $42.9 \%$ (95\% CI: $35.6 \%-50.6 \%$ ), and 50.9\% (95\% CI: 43.3\%-58.5\%), respectively. The addition of other predictors not included in the final model did not significantly improve the performance of the CLIF-C AD score (Supplementary Table 2).

\section{Validation of the CLIF-ACLFS}

The comparative C-index estimates for the internal and external validation cohorts are shown in Table 3 . In the 500 CANONIC patients without ACLF randomly selected for the internal validation analysis, the predictive ability of CLIF-ACLF score at each main time-point was significantly better than those corresponding to MELD, MELD-Na and CP scores. The C-index estimates for all the scores at all time-points were similar to those obtained from the whole population of CANONIC patients.

The external validation analysis included 225 patients without ACLF. CLIF-C AD score improved the predictive ability of the other 3 scores, although this improvement was statistically significant only for 90-day mortality. This is probably due to the fact that the estimates have been obtained with a limited sample size. Moreover, all C-index estimates, in particular those obtained for the CLIF-C AD score, were coherent and similar to those obtained for the whole CANONIC population and for the internal validation sample.

\section{Sequential use of the CLIF-ACLFS}

Table 4 reports the C-index estimates for the CLIF-ADs and MELDs, computed at enrollment and at $48 \mathrm{~h}, 3-7$ days, and 815 days after enrollment, in the 344 CANONIC study patients with $\mathrm{AD}$ at enrollment and with post-enrollment follow-up clinical and laboratory data. When used sequentially, CLIF-C ADs improved its predictive performance, with statistically significant

Table 3. Predictive discrimination ability of CLIF-C AD score as compared to MELD and MELD-Sodium. Patients without ACLF at enrolment in the internal and externa validation databases.

\begin{tabular}{|c|c|c|c|c|}
\hline & $\begin{array}{l}\text { CLIF-C AD score } \\
\text { C-index }(95 \% \mathrm{CI})\end{array}$ & $\begin{array}{l}\text { Child-Pugh score } \\
\text { C-index }(95 \% \mathrm{Cl})\end{array}$ & $\begin{array}{l}\text { MELD score } \\
\text { C-index }(95 \% \mathrm{CI})\end{array}$ & $\begin{array}{l}\text { MELD-Na score } \\
\text { C-index }(95 \% \mathrm{CI})\end{array}$ \\
\hline \multicolumn{5}{|c|}{ Internal validation database. Random sample of CANONIC patients $(N=500)$} \\
\hline 90-day mortality & $0.726(0.668-0.784)$ & $0.608(0.534-0.682)$ & $0.631(0.562-0.700)$ & $0.652(0.584-0.720)$ \\
\hline$p$ value vs. CLIF-C ADs* & & $<0.001$ & $<0.001$ & 0.002 \\
\hline 180-day mortality & $0.700(0.646-0.753)$ & $0.606(0.544-0.669)$ & $0.611(0.552-0.670)$ & $0.633(0.573-0.692)$ \\
\hline$p$ value vs. CLIF-C ADs* & & $<0.001$ & $<0.001$ & $<0.001$ \\
\hline$p$ value vs. CLIF-C ADs* & & 0.003 & $<0.001$ & 0.002 \\
\hline \multicolumn{5}{|c|}{ External validation database $(\mathbf{N}=225)$} \\
\hline 90-day mortality & $0.744(0.629-0.859)$ & $0.633(0.523-0.742)$ & $0.647(0.545-0.749)$ & $0.711(0.630-0.793)$ \\
\hline$p$ value vs. CLIF-C ADs* & & 0.043 & 0.013 & 0.299 \\
\hline 180-day mortality & $0.683(0.590-0.775)$ & $0.627(0.541-0.713)$ & $0.617(0.530-0.704)$ & $0.654(0.576-0.733)$ \\
\hline$p$ value vs. CLIF-C ADs* & & 0.609 & 0.630 & 0.956 \\
\hline$p$ value vs. CLIF-C ADs* & & 0.753 & 0.306 & 0.548 \\
\hline
\end{tabular}

${ }^{*} p$ values from the Integrated Discriminating Improvement (IDI) statistics test. 
Table 4. Sequential use of the CLIF-C AD and MELD score to predict mortality in CANONIC patients without ACLF at enrollment and post-enrollment clinical and laboratory follow-up.

\begin{tabular}{llllll}
\hline & \multicolumn{3}{c}{ CLIF-C AD score } & & \multicolumn{2}{c}{ MELD score } \\
\cline { 2 - 5 } \cline { 5 - 6 } & C-index $(95 \% \mathrm{Cl})$ & $p$ value vs. baseline & & C-index (95\% Cl) & $p$ value vs. baseline \\
\hline Scores at enrollment $(\mathrm{N}=344)$ & $0.644(0.585-0.702)$ & & $0.591(0.535-0.647)$ & \\
Scores after 48 hours $(\mathrm{N}=236)$ & $0.667(0.611-0.734)$ & 0.443 & $0.579(0.515-0.642)$ & 0.689 \\
Scores after 3-7 days $(\mathrm{N}=273)$ & $0.666(0.605-0.728)$ & 0.463 & $0.612(0.549-0.676)$ & 0.484 \\
Scores after 8-15 days $(\mathrm{N}=165)$ & $0.714(0.644-0.784)$ & 0.020 & $0.608(0.526-0.689)$ & 0.571 \\
\hline
\end{tabular}

${ }^{*} p$ value $v s$. score estimates at enrolment.

results at 1-year. These findings were not observed with regard to MELD.

\section{Discussion}

The present study used the data acquired in the CANONIC study $[3,5]$ to focus on a group of patients with acutely decompensated cirrhosis with no ACLF, and has generated a validated new score, the CLIF-AD score, which can be used to prognosticate survival of these patients on the medium (3-6 months) and long term ( 1 year). The score also shows that it retains its accuracy when it is updated sequentially suggesting that the score responds to potential clinical interventions that may affect outcome. The CLIF-C AD score was validated both externally and internally with a resampling from the CANONIC dataset, since imbalances found between the derivation and the external validation sets might have affected the external validation results. With further validation, this score may be useful in conjunction with the CLIF-C Organ failure score (CLIF-C OFs) and the CLIF-C ACLF score to define the prognosis of a cirrhotic patient who is hospitalised with AD of cirrhosis [3]. An algorithm for how this score may be used in combination with the CLIF-C ACLF score is illustrated in Fig. 4. In order to use this score effectively, scores should be updated on a daily basis. An online calculator and application are available at www.clifconsortium.com. Thus, the CLIF-C OF score should be applied to all cirrhotic patients that are admitted

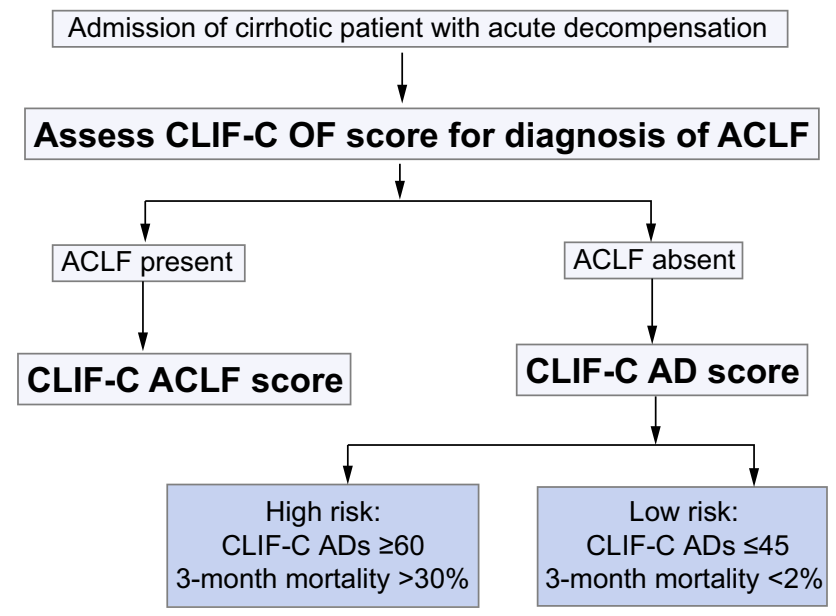

Fig. 4. Proposed algorithm for the use of EASL-CLIF Consortium predictive scores for ACLF and non-ACLF patients. The 3-month mortality of the low-risk group is $1.8 \%$ whereas the risk of mortality of the high-risk group is $31.3 \%$. to the hospital with an AD. This will allow the classification of patients into a group that has or has not got ACLF, which is referred to as the $A D$ group. In those with ACLF, the 3-month mortality is about $51 \%$ and the CLIF-C ACLF score has been validated to provide prognostic information in these patients $[3,5]$. In those without ACLF, the 3-month mortality is about $13 \%$ and we propose that the CLIF-C ADs should be applied to these patients to provide prognostic information [5]. The score allowed the identification of a large group of patients (274, $27 \%$ ) with a very low 3 -month mortality (CLIF-C ADs $<45$, survival rate $98.2 \%$ ) suggesting that these patients could be discharged early, which would provide significant healthcare savings and reduce risk of nosocomial infections and distress to the patient and the family [6]. On the other hand, a high-risk group was also identified (163 patients, $16 \%$; CLIF-C ADs $\geqslant 60$; 3-month survival rate: $68.7 \%$, showing a 90 -day mortality rate similar to that reported by Moreau et al. [5] in patients with ACLF grade 1. These patients represent a unique population to assess new early interventions to prevent progression to multi-organ failure and death, or selected for work up early for liver transplantation. This hypothesis will need to be tested in large prospective studies.

The general principles behind developing the CLIF-C ADs were to ensure that it would be simple to use and provide prognostic information using variables that can be easily available to the clinicians who first see the patients at the time of hospital admission. The score should be able to provide figures estimating risk of mortality and also allow an easy stratification of patients into high and low risk groups. Most importantly, the new score should provide prognostic information that improves upon the best scoring systems available, the MELD, MELD-Na and the CP score [3].

As already observed for ACLF patients [17], the factors independently associated with poor prognosis in AD patients can be also considered under the PIRO concept. 'P' in PIRO refers to Predisposition. It is important to note that only age had an important weight in predicting mortality as it was also observed in the CLIFC ACLF score. The etiology of the underlying liver disease did not have discriminatory ability. It is notable that the present series is representative of typical patients in the West World with relative paucity of patients with Hepatitis B virus infection. Validation in this cohort will be required in future studies. 'I' refers to Injury. The presence or the absence of a precipitating event or the type was not associated with risk of mortality. 'R' refers to Response. As in the CLIF-C ACLF score [3], the white cell count was independently associated with poor outcome indicating that the higher risk patients have a more marked systemic inflammatory response providing perhaps insights into potential future targets of therapy. The white cell count remained a significant poor prognostic marker independently from the presence of alcoholic liver disease or infection (data not shown). 'O' refers to Organs. 


\section{JOURNAL OF HEPATOLOGY}

Consistently with previous studies, it was not surprising that serum sodium, serum creatinine and INR, the last two variables representing organ/system function, showed predictive ability. These variables are already components of the MELD-Na score, which have been studied extensively providing another validation of the approach used in the present study [7-9]. Therefore, our data suggest that both age and inflammatory response, the latter measured using the white cell count, add discrimination ability to organ function predictors, explaining the higher accuracy of CLIF-C ADs with respect to other liver scores. Through the final model including these 5 variables, we developed a new score (CLIF-C ADs) ranging between 0 and 100 points, which accurately predicted the risk of mortality. In order to take into account the potential to change the outlook of patients with interventions such as treatment of the underlying disease, precipitating factors, modifying inflammatory response or improving organ function, we assessed the score for sequential measurements by showing that the mid- and long-term predictive accuracy of the CLIF-ADs is retained or improves when measured again at $48 \mathrm{~h}, 3-7$ days, and 8-15 days.

The next step was to compare the performance of this score against the current gold standards, the MELD, MELD-Na, and the $\mathrm{CP}$ scores. Comparison between the CLIF-C AD and MELD, MELD-Na and CP score was assessed in three different ways, which suggested the superiority of the CLIF-C ADs over the other scoring systems. First, the AUROC analysis clearly showed that the CLIF-C AD score was significantly more accurate in predicting 3-month mortality compared with MELD, MELD-Na, and CP score. Second, the observed $v s$. predicted mortality rates indicated by the concordance index in the derivation cohort showed that CLIF-C AD score was significantly more accurate in predicting 3month, 6-month and 12-month mortality than MELD, MELD-Na, and $\mathrm{CP}$ score. The accuracy of these observations was confirmed in the internal validation cohort. Concordance indices confirmed better performance of the CLIF-C AD score in the external validation data, which was statistically significant at predicting 3-month mortality against MELD and CP score. It is possible that the relatively small sample size and the relatively low event rate of the validation cohort account for the lack of statistical significance at other time-points. Third, the CLIF-C AD score improved the performance of these other scores by $8-15 \%$ being most relevant at predicting 3-month mortality in both derivation and validation cohorts.

The assessment of the percentage effect-size estimates of each variable explains the better performance of the CLIF-C AD score compared with the MELD and MELD-Na results. The effect size of age, which is not included in MELD or MELD-Na scores, was similar to that of creatinine and bilirubin, which are important components of both scores. Additionally, creatinine and especially bilirubin showed a limited effect-size for mortality, probably due to the absence of high-risk patients (with ACLF) in this analysis population. Finally, white cell count and serum sodium, CLIF-C ADs components that showed the highest effect-size for mortality, are absent in CP and MELD, while white cell count is not included in MELD-Na score.

In our study, the C-index values observed for MELD and MELD-Na scores (ranging from 0.58 to 0.71 ) are among the lowest reported by other investigators [18-24]. This is probably related to the difference between our study and the other investigations in patients' characteristics and times of score assessment. In most investigations, the MELD and/or MELD-Na were assessed in patients with any type of liver disease at the time of enrollment on the waiting list for liver transplantation. In contrast, in our study all patients had cirrhosis and scores were obtained in nearly all cases at the time of admission to the hospital for an $\mathrm{AD}$ of cirrhosis. Patients with an $\mathrm{AD}$ of cirrhosis are extremely unstable and they may improve or worsen within a few days after admission. Therefore, it is not surprising that, in our study, MELD and MELD-Na scores showed lower discrimination abilities as compared to other studies, in which scores were assessed in patients with more stable conditions. For instance, among the 1016 patients included in the current analysis, 115 (11.3\%) developed an ACLF within 28 days after study enrollment (data not shown). Approximately $50 \%$ of these patients died within 3 months after enrollment. On the other hand, in many other patients, renal and hepatic functions improved rapidly following standard medical treatment.

Despite the improvement of the prognostic accuracy observed with the CLIF-C AD score over the MELDs, MELD-Nas and CPs, in our results a significant proportion of incorrect predictions $(26 \%$ for 90-day mortality) is still present, thus indicating the need for further studies on additional and/or more accurate prognostic markers in these patients.

In summary, the data presented in this paper support a validated, new scoring system using simple clinical variables that can be updated sequentially to determine the mortality of patients who present with AD of cirrhosis and have no ACLF. This score performs significantly better than existing scoring systems. When combined with the CLIF-C OF and the CLIF-C ACLF score, the outlook of all patients with cirrhosis that require hospital admission with $\mathrm{AD}$ can be mapped according to the proposed algorithm and classified in low and high-risk cohorts. The proposed algorithm should be validated prospectively in large studies.

\section{Financial support}

The CLIF Consortium is supported by an unrestricted grant from Grifols. Rajiv Jalan is supported by a comprehensive biomedical research center, UK grant; Richard Moreau was supported by an INSERM-APHP fellowship and Jonel Trebicka by grants from the Deutsche Forschungsgemeinschaft (SFB TRR57 project 18).

PG received a grant from the Fondo de Investigación Sanitaria Carlos III - co financed by FEDER: FIS_PI12/330.

\section{Conflict of interest}

Rajiv Jalan received research funding from Vital Therapies, has served on Scientific Advisory Board for Conatus Pharma, and received lecture fees from Gambro and has on-going research collaboration with Gambro, Grifols and is the Principal Investigator of an Industry sponsored study (Sequana Medical). He is also the inventor of a drug, L-ornithine phenylacetate, which UCL has licensed to Ocera Therapeutics. Faouzi Saliba has received speaker honorarium and/or grant research from Novartis, Astellas, Roche, Merck Sharp \& Dohme, Pfizer, Gambro and Vital Therapies. Pere Ginès has received speaker honorarium and research funding from Grifols, served on the scientific advisory board for Ferring and Sequana and received research funding from Sequana. Alexander Gerbes has served as a consultant to CSL Behring. 


\section{Research Article}

Stefan Zeuzem has served as a consultant to Abbott, Achillion, AstraZeneca, BMS, Boehringer Ingelheim, Gilead, Janssen Cilag, Merck, Novartis, Presidio, Roche, Santaris, Vertex. Vicente Arroyo has received grant and research support from Grifols. All other authors declare that they have no conflicts of interest.

\section{Authors' contributions}

RJ, FS, VA: study design, data evaluation, manuscript writing and final review; MP, AA: study design, statistical analysis, manuscript writing and review; RM, PG, MB: study design and final manuscript review; JF, PHF, RS, RM, PC, FD, PA, CA, WL, JT, DS, SZ, TG, AG, JW: patient recruitment, data collection and final manuscript review.

\section{Disclosures}

\section{The EASL-CLIF Consortium}

It is a network of 63 European university hospitals, aimed at stimulating research on pathophysiology, diagnostic and treatment on Chronic Liver Failure. During the period 2009-2012 the EASL-CLIF Consortium had received unrestricted grants from Grifols and Gambro. Grifols has prolonged its unrestricted grant for an additional period of four years. There is no other support for the Consortium. The Fundació Clinic, a foundation ruled by the Hospital Clinic and University of Barcelona, administers the EASL-CLIF Consortium grants. Vicente Arroyo (Chairman), Mauro Bernardi (Vice-Chairman), and members of the Steering Committee have no relationship with Grifols or Gambro other than conferences at international meetings (from which they may receive an honorarium) or as investigators on specific projects unrelated to the consortium. Up to now the EASL-CLIF Consortium has not performed any study promoted by pharmaceutical companies. The scientific agenda of the EASL-CLIF Consortium and the specific research protocols are made exclusively by the Steering Committee members without any participation of pharmaceutical companies.

\section{Appendix. CANONIC study investigators of the EASL-CLIF consortium (alphabetical order)}

1. Patricia Aguilar Melero, Hospital Universitario Reina Sofía. Centro de Investigación Biomédica en Red Enfermedades Hepáticas y Digestivas (CIBERehd). Instituto Maimónides de Investigación Biomédica de Córdoba. (IMIBIC) Córdoba, Spain.

2. Agustin Albillos, Servicio de Gastroenterología, Hospital Universitario Ramón y Cajal, Universidad de Alcalá, Centro de Investigación Biomédica en Red Enfermedades Hepáticas y Digestivas (CIBERehd), Madrid, Spain.

3. Rafael Bañares, Hospital General Universitario Gregorio Marañón, Instituto de Investigación Sanitaria Gregorio Marañón (IiSGM), Centro de Investigación Biomédica en Red Enfermedades Hepáticas y Digestivas (CIBERehd), School of Medicine. Universidad Complutense. Madrid, Spain.
4. Daniel Benten, Department of Gastroenterology and Hepatology, University Medical Center Hamburg-Eppendorf, Hamburg, Germany.

5. Massimo Bocci, Department of Gastroenterology and Hepato-Pancreatology, Erasme Hospital, Université Libre de Bruxelles, Brussels, Belgium.

6. Paolo Caraceni, Semeiotica Medica, Policlinico S. OrsolaMalpighi Department of Medical and Surgical Sciences University of Bologna, Bologna, Italy.

7. María-Vega Catalina, Hospital General Universitario Gregorio Marañón, Instituto de Investigación Sanitaria Gregorio Marañón (IiSGM), Centro de Investigación Biomédica en Red Enfermedades Hepáticas y Digestivas (CIBERehd), Madrid, Spain.

8. Jun Liong Chin, Liver Unit, St Vincent's University Hospital, Dublin, Ireland.

9. Minneke J. Coenraad, Leiden University Medical Centre, Department of Gastroenterology-Hepatology, Leiden, The Netherlands.

10. Mar Concepción, Department of Gastroenterology, Hospital de la Santa Creu i Sant Pau, Barcelona, Universitat Autònoma de Barcelona, Barcelona, Spain.

11. Audrey Coilly, Centre Hépato-Biliaire, Hôpital PaulBrousse, Assistance Publique-Hôpitaux de Paris, Villejuif, France.

12. Livia Dorn, Department of Gastroenterology and Hepatology, Innsbruck Medical University, Innsbruck, Austria.

13. Carme Deulofeu, Data Management Centre, CLIF Consortium, Hospital Clinic, Barcelona, Spain.

14. Laure Elkrief, Service d'Hépatologie, Hôpital Beaujon, Assistance Publique Hôpitaux de Paris, Clichy; Inserm U773, Centre de Recherche Biomédicale Bichat-Beaujon CRB3, Clichy and Paris; and Université Paris Diderot-Paris 7, Paris, France.

15. Javier Fernandez, Liver Unit, Hospital Clinic, University of Barcelona, CLIF Consortium Centro de Investigación Biomédica en Red Enfermedades Hepáticas y Digestivas (CIBERehd), Barcelona, Spain.

16. Elisabet Garcia, Data Management Centre, CLIF Consortium, Hospital Clinic, Barcelona, Spain.

17. Angelo Gatta, Department of Medicine, University of Padova, Padova, Italy.

18. Ludmila Gerber, Department of Medicine I, JW Goethe University Hospital, Frankfurt, Germany.

19. Andrea de Gottardi; University Clinic of Visceral Surgery and Medicine of Berne, Berne, Switzerland.

20. Isabel Graupera; Liver Unit, Hospital Clinic, University of Barcelona, Centro de Investigación Biomédica en Red Enfermedades Hepáticas y Digestivas (CIBERehd), Barcelona, Spain.

21. Henning Grøenbæk, Department of Medicine V, Unit of hepatology and gastroenterology,Aarhus University Hospital, Aarhus, Denmark.

22. AnneKristin Hausen; Department of Internal Medicine I, University Hospital of Bonn, Bonn, Germany.

23. Corinna Hopf, Liver Center Munich, Department of Medicine 2, Klinikum der LMU München-Grosshadern, Munich, Germany.

24. Stine Karlsen, Department of Medicine V, Unit of Hepatology and Gastroentorology, Aarhus University Hospital, Aarhus, Denmark. 
25. Ansgar W. Lohse Department of Gastroenterology and Hepatology, University Medical Center HamburgEppendorf, Hamburg, Germany.

26. Caterina Maggioli, Semeiotica Medica, Policlinico S. OrsolaMalpighi Department of Medical and Surgical Sciences University of Bologna, Bologna, Italy.

27. Daniel Markwardt, Liver Center Munich, Department of Medicine 2, Klinikum der LMU München-Grosshadern, Munich, Germany.

28. Javier Martinez, Servicio de Gastroenterología, Hospital Universitario Ramón y Cajal, Madrid, Spain.

29. Alfredo Marzano; Division of Gastroenterology and Hepatology, San Giovanni Battista Hospital. University of Turin, Turin, Italy.

30. Manuel de la Mata García Hospital Universitario Reina Sofía. Centro de Investigación Biomédica en Red Enfermedades Hepáticas y Digestivas (CIBERehd). Instituto Maimónides de Investigación Biomédica de Córdoba. (IMIBIC), Córdoba, Spain.

31. P. Aiden McCormick, Liver Unit, St Vincent's University Hospital, Dublin, Ireland.

32. Francisco Mesonero, Servicio de Gastroenterología, Hospital Universitario Ramón y Cajal, Madrid, Spain.

33. José Luis Montero Álvarez, Hospital Universitario Reina Sofía. Centro de Investigación Biomédica en Red Enfermedades Hepáticas y Digestivas (CIBERehd). Instituto Maimónides de Investigación Biomédica de Córdoba. (IMIBIC), Córdoba, Spain

34. Rajeshwar P Mookerjee, Institute of Liver and Digestive Health, Liver Failure Group, Royal Free Campus, London, United Kingdom.

35. Filippo Morando, Department of Medicine, University of Padova, Padova, Italy.

36. Christophe Moreno, Department of Gastroenterology and Hepato-Pancreatology, Erasme Hospital, Université Libre de Bruxelles, Brussels, Belgium.

37. Bernhard Morrell; University Clinic of Visceral Surgery and Medicine of Berne, Berne, Switzerland.

38. Christian Mortensen Department of Gastroenterology, Hvidovre Hospital, University of Copenhagen, Copenhagen, Denmark.

39. Frederik Nevens, Department of Liver and Biliopancreatic Diseases, University Hospital Gasthuisberg, KU Leuven, Leuven, Belgium.

40. Markus Peck-Radosavljevic, Department of Gastroenterology and Hepatology, Medical University of Vienna, Vienna, Austria.

41. Gustavo Pereira, Liver Unit, Hospital Clinic, University of Barcelona, Centro de Investigación Biomédica en Red Enfermedades Hepáticas y Digestivas (CIBERehd), Barcelona, Spain.

42. Alessandro Risso; Division of Gastroenterology and Hepatology, San Giovanni Battista Hospital, University of Turin, Turin, Italy.

43. Mario Rizzetto; Division of Gastroenterology and Hepatology, San Giovanni Battista Hospital, University of Turin, Turin, Italy.

44. Antonietta Romano, Department of Medicine, University of Padova, Padova, Italy.

\section{JOURNAL OF HEPATOLOGY}

45. Didier Samuel, Centre Hépato-Biliaire, Hôpital Paul Brousse, Assistance Publique-Hôpitaux de Paris, Villejuif, France.

46. Tilman Sauerbruch, Department of Internal Medicine I, University Hospital of Bonn, Bonn, Germany.

47. Macarena Simon-Talero. Servicio de Hepatologia, Hospital Vall d'Hebron, Universitat Autònoma de Barcelona, Centro de Investigación Biomédica en Red Enfermedades Hepáticas y Digestivas (CIBERehd), Barcelona, Spain.

48. Elsa Solà-Vergés, Liver Unit, Hospital Clinic, University of Barcelona, Centro de Investigación Biomédica en Red Enfermedades Hepáticas y Digestivas (CIBERehd), Barcelona, Spain.

49. Pablo Solis-Muñoz, Institute of Liver Studies and the Cellular Biology of Inflammation, King's College London, London, United Kingdom.

50. German Soriano, Department of Gastroenterology, Hospital de la Santa Creu i Sant Pau, Barcelona, Universitat Autònoma de Barcelona, Centro de Investigación Biomédica en Red Enfermedades Hepáticas y Digestivas (CIBERehd), Instituto de Salud Carlos III, Barcelona, Spain.

51. Jan Sperl, Department of Hepatogastroenterology, Institute for Clinical and Experimental Medicine, Prague, Czech Republic.

52. Walter Spindelboeck Division of Gastroenterology and Hepatology, Department of Internal Medicine, Medical University of Graz, Graz, Austria.

53. Rudolf Stauber, Division of Gastroenterology and Hepatology, Department of Internal Medicine, Medical University of Graz, Graz, Austria.

54. Christian Steib, Liver Center Munich, Department of Medicine 2, Klinikum der LMU München-Grosshadern, Munich, Germany.

55. Dominique Valla, Service d'Hépatologie, Hôpital Beaujon, Assistance Publique Hôpitaux de Paris, Clichy; Inserm U773, Centre de Recherche Biomédicale Bichat-Beaujon CRB3, Clichy and Paris; and Université Paris Diderot-Paris 7, Paris, France.

56. Len Verbeke, Department of Liver and Biliopancreatic Diseases, University Hospital Gasthuisberg, KU Leuven, Leuven, Belgium.

57. Hans Van Vlierberghe, Department of Gastroenterology and Hepatology, Ghent University Hospital, Ghent, Belgium.

58. Wolfgang Vogel, Department of Gastroenterology and Hepatology, Innsbruck Medical University, Innsbruck, Austria.

59. Henninge Wege, Department of Gastroenterology and Hepatology, University Medical Center Hamburg-Eppendorf, Hamburg, Germany.

60. Tania Welzel, Department of Medicine I, JW Goethe University Hospital, Frankfurt, Germany.

61. Chris Willars, Liver Intensive Care Unit, King's College Hospital, London, United Kingdom.

62. Maria Yago Baenas, Institute of Liver and Digestive Health, Liver Failure Group, Royal Free Campus, London, United Kindgom.

63. Giacomo Zaccherini, Semeiotica Medica, Policlinico S. Orsola-Malpighi Department of Medical and Surgical Sciences University of Bologna, Bologna, Italy. 


\section{Research Article}

\section{Supplementary data}

Supplementary data associated with this article can be found, in the online version, at http://dx.doi.org/10.1016/j.jhep.2014.11. 012.

\section{References}

[1] Jalan R, Gines P, Olson JC, et al. Acute-on chronic liver failure. J Hepato 2012;57:1336-1348.

[2] Jalan R, Yurdaydin C, Bajaj JS, et al. Toward an improved definition of acuteon-chronic liver failure. Gastroenterology 2014;147:4-10.

[3] Jalan R, Saliba F, Pavesi M, et al. Development and validation of a prognostic score to predict mortality in patients with acute on chronic liver failure. J Hepatol 2014;61:1038-1047.

[4] Jalan R, Williams R. Acute-on-chronic liver failure: pathophysiological basis of therapeutic options. Blood Purif 2002;20:252-261.

[5] Moreau R, Jalan R, Gines P, et al. Acute-on-chronic liver failure is a distinct syndrome developing in patients with acute decompensation in cirrhosis. Gastroenterology 2013;144:1426-1437.

[6] Olson JC, Wendon JA, Kramer DJ, et al. Intensive care of the patient with cirrhosis. Hepatology 2011;54:1864-1872.

[7] Kamath PS, Kim WR. The model for end-stage liver disease (MELD). Hepatology 2007;45:797-805.

[8] Kim WR, Biggins SW, Kremers WK, et al. Hyponatremia and mortality among patients on the liver-transplant waiting list. $N$ Engl $J$ Med 2008;359:1018-1026.

[9] Pugh RN, Murray-Lyon IM, Dawson JL, Pietroni MC, Williams R. Transection of the oesophagus for bleeding oesophageal varices. $\mathrm{Br} \mathrm{J}$ Surg 1973;60:646-649.

[10] Ferreira FL, Bota DP, Bross A, Melot C, Vincent JL. Serial evaluation of the SOFA score to predict outcome in critically ill patients. JAMA 2001;286:1754-1758.

[11] Fine JP, Gray RJ. A proportional hazards model for the subdistribution of a competing risk. J Am Stat Assoc 1999;94:496-509.

[12] Wolbers M, Koller MT, Witteman JC, Steyerberg EW. Prognostic models with competing risks. methods and application to coronary risk prediction. Epidemiology 2009;20:555-561.
[13] Harrell Jr FE, Lee KL, Mark DB. Multivariable prognostic models: issues in developing models, evaluating assumptions and adequacy, and measuring and reducing errors. Stat Med 1996;15:361-368.

[14] Pencina MJ, D'Agostino RB. Overall $C$ as a measure of discrimination in survival analysis: model specific population value and confidence interval estimation. Stat Med 2004;23:2109-2123.

[15] Pencina MJ, D’Agostino Sr RB, D’Agostino Jr RB, Vasan RS. Evaluating the added predictive ability of a new marker: from area under the ROC curve to reclassification and beyond. Stat Med 2008;27:157-172.

[16] Altman DG, Royston P. What do we mean by validating a prognostic model? Stat Med 2000;19:453-473.

[17] Jalan R, Stadlbauer V, Sen S, Cheshire L, Chang YM, Mookerjee RP. Role of predisposition, injury, response and organ failure in the prognosis of patients with acute-on-chronic liver failure: a prospective cohort study. Crit Care 2012;16:R227.

[18] Schepke M, Roth F, Fimmers R, et al. Comparison of MELD, Child-Pugh, and Emory model for the prediction of survival in patients undergoing transjugular intrahepatic portosystemic shunting. Am J Gastroenterol 2003;98:1167-1174.

[19] Chologitas E, Marelli L, Shusang V, et al. A systematic review of the performance of the model for end-stage liver disease (MELD) in the setting of liver transplantation. Liver Transpl 2006;12:1049-1061.

[20] Murad SD, Ray Kim W, de Groen PC. Can the model for end-stage liver disease be used to predict the prognosis in patients with vBudd-Chiari syndrome? Liver Transpl 2007;13:867-874.

[21] Sharma P, Schaubel DE, Sima CS, et al. Re-weighting the model for end-stage liver disease score components. Gastroenterology 2008;135:1575-1581.

[22] Biselli M, Gitto S, GRamenzi A. Six score systems to evaluate candidates with advanced cirrhosis for orthotopic liver transplant: which is the winner? Liver Transpl 2010;16:964-973.

[23] Leise MD, Ray Kim W, Kremers WK. A revised model for end-stage liver disease optimizes prediction of mortality among patients awaiting liver transplantation. Gastroenterology 2011;140:1952-1960.

[24] Biselli M, Dall'agata M, Gramenzi A, et al. A new prognostic model to predict dropout from the waiting list in cirrhotic candidates for liver transplantation with MELD score < 18. Liver Int 2015;35:184-191. 